\title{
Analysis of the dynamic characteristics of two-phase flow based on the
}

\section{technology of acoustic emission}

\section{Lide Fang ${ }^{1, a^{*}}$, Zitong Wen ${ }^{2, b}$, Shitao Zhang ${ }^{3, c}$, Mingming Li ${ }^{4, d}$, Dan Li ${ }^{5, f}$ and Yao}

\author{
Zhang $^{6, f}$ \\ 1,2,4 Hebei University,Baoding,China \\ ${ }^{6} \mathrm{Xi}$ an Jiaotong University,Xi an,China \\ ${ }^{3}$ Institute of measurement and testing technology, Baoding 071000, China

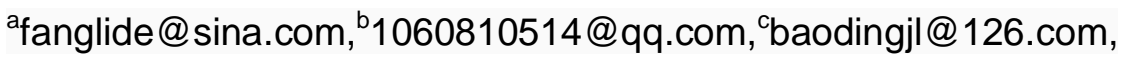 \\ d731260950@qq.com, ${ }^{\text {e3 } 348557812 @ q q . c o m, f f e n g k u a n g y a o @ s i n a . c o m ~}$
}

Keywords:acoustic emission; dynamic characteristic; interphase force; signal extraction Abstract:This paper propose a method to study the dynamic characteristics of two-phase flow though the signals which collected from acoustic emission. With the digital processing to extract the feature of time domain and wavelet energy to analysis. the characteristic of flow. At last, get the relationship of Frg and flow status. Though study the pressure loss of vertical direction get the model of interphase force. Different flow pattern with different characteristic of dynamic of flow. Acoustic emission could be a new method to study the two phrase flow in the future.

\section{Introduction}

At the industrial production, the multiphase flow field has been as an important research branch of petrochemical attention by scholars and experts. Multiphase flow due to the flow sate show its complexity relative to the signal phase flow.Gas liquid two phase flow under the condition of different gas and liquid ratio present different flow patterns and phase holdup. Therefore has brought the difficulty to study. And dynamic characteristics of gas-liquid two phase flow also cause diversity of flow pattern. This paper based on the acoustic emission technology to analysis gas liquid two phase flow state and flow mechanism.Acoustic emission technology is one kind of nondestructive testing, The cause of acoustic emission sensor collected signal is rapid release of energy and a transient elastic wave to detection the defects. Used the unique advantage of gas liquid two phase flow, the non-conected way has no inference to convention type. Real time noise signal acquisition analysis of the two phase flow, gas liquid two phase flow in the process of flow medium will not harmful to probe acquisition signal ${ }^{[1-2]}$.

\section{Noise signal acquisition device}

The experimental platform could conduct oil gas and water three phase flow experiment and test the related parameters of multiphase flow.Using the vallen - system of the company's AMSY - 5, 8 channels of acoustic emission sampling device. AMSY - 5 type acoustic emission system is illustrated below. This experiment uses the sampling rate is $0.6 \mathrm{MHZ}$, sampling point is 524288, experimental probe device adopts four probe acoustic emission signal acquisition, Fig. 1 is noise signal acquisition device ${ }^{[3-4]}$. 


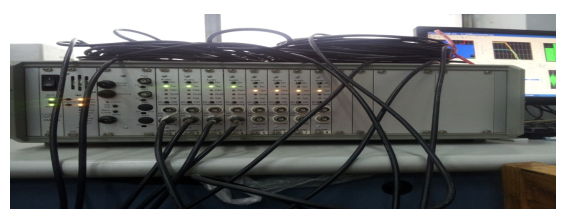

Fig. 1 AMSY - 5 acoustic emission

First, To extract noise signal under different flow patterns, Respectively to extract the bubble flow, stratified flow and annular flow, the experimental conditions was L4g0.03, L0.5g5, L8g80.Time domain diagram as shown in the fig. 3(a-c):

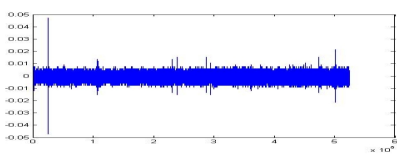

(a )bubble flow

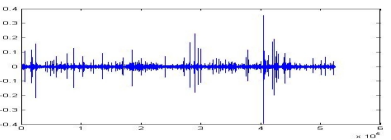

(b) stratified flow

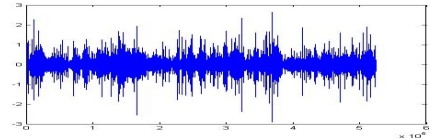

(c) annular flow

Fig.2 Time domain diagram

Through the fig.2(a-c) observed,gas liquid two phase flow under the different flow pattern in the acoustic emission signal noise show completely different signal intensity,characterized with the flow status of fluid inside there is a cause of its big differences ${ }^{[5]}$.

Wavelet decomposition has a wider application in signal analysis and Characteristic parameters of quantitative analysis.In the paper,chose Two-dimensional wavelet as wavelet basis of wavelet decomposition for 8 layers of wavelet decomposition.Exacted each layer of the wavelet coefficient,Finally solved the each layer of decomposition of wavelet coefficient energy for quantitative analysis.Under the typical flow pattern of wavelet decomposition energy specifically shown in the following table 1 :

Table 1 wavelet energy decomposition data tables

\begin{tabular}{|c|c|c|c|c|c|c|c|c|c|}
\hline & \multicolumn{3}{|c|}{ Bubble flow } & \multicolumn{3}{c|}{ Stratified flow } & \multicolumn{3}{c|}{ Annular flow } \\
\cline { 2 - 11 } & L4G0.03 & L8G.003 & L8G0.3 & L05G5 & L2G5 & L4G5 & L8G50 & L8G80 & L10G8 \\
\hline $\mathrm{ca} 1$ & 1.4536 & 1.0847 & 1.2541 & 108.6254 & 70.0698 & 99.5698 & 2896.2985 & 2659.2563 & 3632.2956 \\
\hline $\mathrm{cd} 1$ & 1.689 & 1.2534 & 1.4926 & 128.9541 & 79.1698 & 138.1256 & 3098.5615 & 3705.5241 & 3529.2514 \\
\hline $\mathrm{cd} 2$ & 0.9921 & 0.9141 & 0.8645 & 90.584 & 78.596 & 89.9658 & 2496.5695 & 3400.5487 & 3425.2562 \\
\hline $\mathrm{cd} 3$ & 0.1545 & 0.2009 & 0.1015 & 2.856 & 3.698 & 4.856 & 156.5695 & 199.95461 & 185.6952 \\
\hline $\mathrm{cd} 4$ & 0.0103 & 0.0331 & 0.0298 & 0.9487 & 0.435 & 0.8025 & 23.036956 & 32.0215 & 28.1526 \\
\hline $\mathrm{cd} 5$ & 0.0125 & 0.0185 & 0.0204 & 0.1526 & 0.0895 & 0.1956 & 2.9585 & 3.9562412 & 2.6952 \\
\hline $\mathrm{cd} 6$ & 0.0065 & 0.0090 & 0.0067 & 0.1596 & 0.00135 & 0.3652 & 0.50659 & 0.568565 & 0.495261 \\
\hline
\end{tabular}

According to the above listed the level of test section under the three typical flow pattern of the standard under the point of energy distribution can be observed,first, the energy of noise signal of three different flow pattern distributed in the high frequency part,under bubble flow, the minimum energy is 0.0109 , the maximum is 1.7485 . Stratified flow of energy in the annular flow and bubble flow between the energy range of 0.0165-720.Annular flow, with the increase of gas liquid phase flow energy is obviously showed a trend of larger 3560.Analysis from the pipeline internal flow mechanism, Through wavelet decomposition energy could see the change of gas liquid two phase flow under different flow patterns, Gas-liquid two-phase flow status inside the pipeline change violently, so collected acoustic emission signal energy exists obvious difference. 


\section{Dynamic characteristics analysis}

Through analysis found that internal of gas liquid two phase flow force each other, cause of flow pattern appeared a huge differences and the great different flow state.In the process of the gas phase and liquid phase flow in the pipe,with the gas phase flow,liquid fluid generated interphase forces.Expressed in formula: $\triangle \mathrm{Pv}=\triangle \mathrm{Pg}+\triangle \mathrm{Pr} \Delta \mathrm{PV}$ is perpendicular to the flow to the measured pressure difference, $\Delta \mathrm{PGr}$ for gas-liquid two pictures of a pressure differential and $\Delta \mathrm{Pb}$ for gas and liquid two phases is perpendicular to the flow direction of the pressure difference between the applied force.

Experiment were conducted in points:0.05Mpa,0.1Mpa,0.15Mpa,Each pressure point at the beginning of the stratified flow, along with the increase of gas flow, make the transition to annular flow gradually.Fig. 3 is interphase entrainment reaction and gas phase froude number Frgl relation curve:

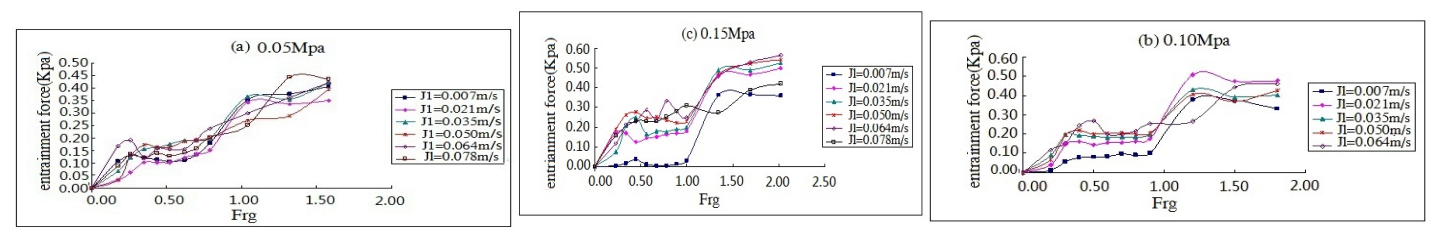

Fig. 3 Entrainment reaction

Fig. 3 is at three points a,b and c,with the increase of gas phase froude number Frg, the change trend of entrainment forces are basically the same.Under the same liquid apparent Jl,when Frg $=0$, the two phase flow is stratified flow, Gas-liquid two phase entrainment reaction basic does not exist.With the increase of Frg,Entrainment force rendering increases and then leveled off and remain near a particular value fluctuation trend,Due to generate upward gas entrainment force, make the liquid interface to volatility, flow state into wavy stratified flow. When Frg $=0.89$,perpendicular to the volume of alternate with entrainment force has increased dramatically.

\section{Summary}

Through the noise signal collected by the pipe, analyzed the cause of difference signal,it was producted by the two phase flow interphase forces. When the gas phase flow rate is smaller, The dynamic characteristics is relatively stable, appears as bubble flow and stratified flow. When the gas flow and liquid flow increases, The dynamic characteristics of significant change,appears as annular flow. Two-phase flow noise signals were collected by means of time domain analysis and extraction of signal energy of wavelet analysis, wavelet energy reflects the size of the two phase flow fluid internal energy and kinetics characteristics. Distinct the flow state and the dynamic characteristics under different flow pattern,get the whole process from the bubble flow to annular flow in big difference.

\section{References}

[1] Guo liejin, Li guangjun, Chen xuejun. Study on the pattern of Oil-gas two phrase about Horizontal spiral pipe[J]. Journal of engineering thermoplastic.1999,17 (4) 472-476.(in Chinese)

[2]Gui-Bo Zheng, Ning-De Jin, Xiao-hui Jia, Peng-Ju Lv and Xing-Bin Liu, Gas-liquid two phase flow measurement method based on combination instrument of turbine flowmeter and conductance 
sensor[J], Int. J.Multiphase Flow, 2008, 34: 1031-1047.(in Chinese)

[3] Marco D, Vittorio F and Domenico S, A sensor system for oil fraction estimation in a two phase oil-water flow[J], Procedia Chemistry, 2009, 1: 1247-1250.

[4] César M S, Cláudio M P, Roberto S and Luis E B, 2010, Flow regime identification and volume fraction prediction in multiphase flows by means of gamma-ray attenuation and artificial neural networks[J], Progress in Nuclear Energy, 2010, 52: 555-562.

[5] H. M. Letel, J. C. Schouten, R. Krishna, C. M. Van den Bleek, Characterization of regimes and regime transitions in bubble columns by chaos analysis of pressure signals[J], Chemical Engineering Science, 1997, 52(24): 4447-4459

[6]Chen Xuejun, Multiphase flow Thermophysics [M], Xi'an Jiao Tong University Press, 2005.(in Chinese) 